\title{
Survival-Prolonging Effects of a Traditional Chinese Medicine Pancreas-Cleansing, Clog-Removing (Qiyi Huaji) Formula on Pancreatic Cancer and Mechanism Study $\mathrm{Yi} \mathrm{Xu}{ }^{1}, \mathrm{~S} \mathrm{Xu}^{2}, \mathrm{Y} \mathrm{Cai}^{2}, \mathrm{LLiu}^{1}$
}

\begin{abstract}
Objectives: To evaluate the performance of a traditional Chinese medicine pancreas-cleansing, clog-removing (Qiyi Huaji, QYHJ) formula in prolonging survival of pancreatic cancer (PC), stabilizing tumor growth, and inhibiting tumor cell proliferation.
\end{abstract}

Methods: A subcutaneous mouse xenograft model was established by using human PC cell line SW1990, from which tumor masses were collected after they were euthanasized. Then nude mice were in situ inoculated with the tumors, which were randomly into two batches, with three groups [a control group treated with normal saline, a QYHJ group and a gemcitabine (Gem) group] in each batch. The survival, maximum tumor diameter and transverse diameter of the mice were recorded. After $24 \mathrm{~h}, 48 \mathrm{~h}$ and $72 \mathrm{~h}$ of drug interventions, the optical densities at $490 \mathrm{~nm}$ were measured.

Results: The QYHJ group had significantly longer survival and higher proliferation inhibition rate than those of the Gem and control groups $(\mathrm{P}<0.01)$, but the QYHJ group and Gem group had similar tumor volume and weight $(\mathrm{P}>0.05)$.

Conclusion: QYHJ formula effectively prolonged the survival of PC and inhibited the proliferation of tumor cells, thus being worthy of promotion in clinical practice.

Keywords: Gemcitabine, pancreatic cancer, proliferation inhibition rate, tumor-bearing survival

From: ${ }^{1}$ Department of Integrated Traditional Chinese and Western Medicine, Fudan University Shanghai Cancer Center, Shanghai 2000322, PR China and ${ }^{2}$ College of Basic Medical Science, Zhejiang Chinese Medical University, Hangzhou 310053, P R China.

Correspondence: Dr L Liu, Department of Integrated Traditional Chinese and Western Medicine, Fax:+86-21-64175590-3638, email: liulmditcwm@163.com 


\section{INTRODUCTION}

Pancreatic cancer (PC), as one of the common diseases threatening human life, is a highly malignant, refractory tumor of the digestive tract. Over $90 \%$ of PC patients have ductal adenocarcinomas of the glandular epithelium, with the mortality rate as high as $99 \%$ and the 5-year survival rate of less than $5 \%$. The ratio of male patients to female ones is $1.75: 1$. Currently, the survival of PC patients can only be prolonged by radical surgeries, but the postoperative 5-year survival rate is only about $20 \%$. Besides, approximately $90 \%$ of PC patients cannot be well treated because they are diagnosed in the locally advanced stage $(1,2)$. Cheng et al. (3) reported that traditional Chinese medicine showed satisfactory therapeutic effects on PC patients. Traditional Chinese medicine pancreas-cleansing, clog-removing (Qiyi Huaji, QYHJ) formula contains Hedyotis diffusa Willd, Amorphophallus rivieri Durieu, Scutellaria barbata D. Don, Gynostemma pentaphyllum (Thunb.) Makino and Alpinia tonkinensis Gagnep, etc. QYHJ formula can remarkably inhibit the growth of PC, especially that of ductal adenocarcinoma (4). Traditional Chinese medicine has shown particular advantages toward advanced PC (5-7). In addition, compared with systemic chemotherapy alone, combining it with QYHJ formula can evidently prolong the median survival of patients with advanced PC and raise the survival rate $(8,9)$. By using animal and cell experiments simultaneously, we herein aimed to asses the effects of QYHJ formula on extending PC survival, stabilizing tumor growth and suppressing tumor cell proliferation. 


\section{METHODS}

\section{Drugs and animals}

QYHJ formula concentrate $(1.8 \mathrm{~g} / \mathrm{ml})$ was purchased from Jiangyin Tianjiang Pharmaceutical Co., Ltd. Gemcitabine was obtained from Eli Lilly and Company (USA). Male BALB/c (nu/nu) nude mice (4-5 weeks old, 20-30 g) were bought from Shanghai Institute of Materia Medica, Chinese Academy of Sciences. The experimental protocol has been approved by local animal ethics committee.

\section{Cell lines}

Human PC SWI990 cell line and mouse PC Panc 02 cell line were provided by ATCC and MD Anderson Cancer Center respectively.

\section{Main reagents and apparatus}

Gene expression profile microarray was purchased from Shanghai Biostar Gene Chip Co., Ltd. Cy3-dCTP and cy5-dCTP were bought from Amersham Pharmalia. Reverse transcriptase was obtained from Gibco BRL. GeneQuant pro DNA analyzer was provided by Biochrom, and microplate reader was purchased from Sunrise.

\section{Animal experiment}

A subcutaneous mouse xenograft model was established by using human PC cell line SW1990, from which tumor masses were collected after they were euthanasized. Then nude mice were in situ inoculated with the tumors, which were randomly into two batches, with three groups in each batch.

First batch: Three groups were administrated with normal saline (control), QYHJ formula (QYHJ) and gemcitabine (Gem) respectively until their deaths to observe the survival times. 
Second batch: Three groups were administrated identically. Meanwhile, a non-treatment group was also set. All the groups were administrated for three weeks, and the maximum tumor diameter and transverse diameter were measured every three days to estimate tumor volume (V). They were executed one day after the last drug interventions, from which tumor masses were isolated to weigh them and to calculate inhibition rate.

\section{Cell experiment}

Human SW1990 cells were divided into a blank control group, a QYHJ formula (aqueous extract) group and a gemcitabine group (positive control), and cultured for $24 \mathrm{~h}, 48 \mathrm{~h}$ and 72 h. Afterwards, culture medium in 96-well plates was replaced with fresh one containing MTT (50 g/L DMSO). After another $4 \mathrm{~h}$ of culture at $37^{\circ} \mathrm{C}$, the culture medium was removed again, into which was added $200 \mu \mathrm{L}$ of DMSO to completely dissolve solids. Then the optical density (OD) at $490 \mathrm{~nm}$ was determined by MRXII absorption spectrometer to calculate the cell proliferation inhibition rate.

\section{RESULTS}

\section{Survival of tumor-bearing mice}

Different mouse groups were administrated until their deaths, with their survival observed and recorded. The survival curves of all the groups are shown in Fig. 1.

The median survival times of the control, QYHJ and Gem groups were 28.50, 39.00 and 21.00 days respectively. Compared with the control group, the survival of the QYHJ group was significantly prolonged $(\mathrm{P}<0.01)$, whereas that of the Gem group was similar $(\mathrm{P}>0.05)$. 
The QYHJ group survived significantly longer than the Gem group did $(\mathrm{P}<0.001)$.

\section{Inhibition of tumor cell growth}

The maximum diameters and transverse diameters of all mice were measured by a sliding caliper every three days to estimate the tumor volume:

$\mathrm{V}=1 / 2 \times\left[\right.$ diameter $\left.\times(\text { transverse diameter })^{2}\right]$

Tumor growth curves were plotted with the mean value of each measurement (Fig.

2).

As time went by, tumors enlarged at different speeds from the 12th day, following a descending order of control group> QYHJ group> Gem group. In addition, the QYHJ and Gem groups had significantly smaller tumors than the control group $\operatorname{did}(\mathrm{P}<0.05)$, but those of the former two groups were similar $(\mathrm{P}>0.05)$.

\section{Inhibition rate of mouse tumors}

The second batch of mice were killed by cervical dislocation 22 days after administration, from which tumor masses and weighed to estimate the inhibition rate:

Inhibition rate $(\%)=(1-$ mean tumor weight of treatment group/mean tumor weight of non-treatment group) $\times 100 \%$

The inhibition rates on the 22nd day are shown in Fig. 3.

According to Eq. 2, tumor inhibition rate is negatively correlated with the mean tumor weight of treatment group, i.e. the inhibition rate increases with increasing mean tumor weight (reducing therapeutic effects), and vice versa. The control group had the highest tumor weight (poorest therapeutic effect), followed by those of the QYHJ and Gem groups sequentially. The tumor inhibition rates of the three groups were $60.5 \%, 33.2 \%$ and $31.7 \%$ 
respectively, being consistent with the tumor growth curves.

\section{Inhibition rate of cell proliferation}

OD values of different groups at $24 \mathrm{~h}, 48 \mathrm{~h}$ and $72 \mathrm{~h}$ were measured to calculate the inhibition rate of cell proliferation:

Inhibition rate of cell proliferation $=(1-\mathrm{OD}$ of treatment well/OD of control well $) \times$ $100 \% \quad(3)$

The results are exhibited in Fig. 4.

All the inhibition rates increased with rising drug dose. After $24 \mathrm{~h}, 48 \mathrm{~h}$ and $72 \mathrm{~h}$ of treatment with $2 \mathrm{mg} / \mathrm{ml}$ QYHJ formula, the inhibition rates were similar, which, however, sharply increased to over $70 \%$ in the presence of $50 \mathrm{mg} / \mathrm{ml}$ QYHJ formula (72 h: $78.36 \%$ ). As to the Gem group, the inhibition rates increased moderately to $14.07 \%, 13.54 \%, 48.37 \%$ after $24 \mathrm{~h}, 48 \mathrm{~h}$ and $72 \mathrm{~h}$ of treatment respectively (Gem dose: $30 \mu \mathrm{g} / \mathrm{ml}$ ). Therefore, QYHJ formula inhibited cell proliferation significantly more effectively than Gem did $(\mathrm{P}<0.05)$.

\section{DISCUSSION}

PC is one of the prevalent malignant tumors with low recovery rate and poor prognosis, which can now only be cured by surgeries. Nonetheless, most patients cannot be surgically treated because they are diagnosed in the advanced stage. In this study, we intended to demonstrate the effects of a traditional Chinese medicine QYHJ formula on extending PC survival, stabilizing tumor growth and suppressing proliferation of tumor cells by using animal and cell experiments. QYHJ formula effectively prolonged the tumor-bearing survival of mice, which 
significantly exceeded those of the Gem and control groups. Compared with the control group, QYHJ formula and Gem significantly controlled the increase of tumor volume and weight, without showing inter-group differences. Moreover, QYJH formula inhibited the proliferation of tumor cells more satisfactorily than the outcomes of the control and Gem groups. In the QYHJ group, the inhibition rates after $24 \mathrm{~h}, 48 \mathrm{~h}$ and $72 \mathrm{~h}$ of treatment (dose: 2 $\mathrm{mg} / \mathrm{ml}$ ) were similar, which were elevated to over $70 \%$ when $50 \mathrm{mg} / \mathrm{ml}$ QYHJ formula was used.

Regardless, there remain unsolved issues for the use of QYHJ formula in PC treatment. Traditional Chinese medicine cannot allow targeted therapy of PC. Besides, the mechanism of QYHJ formula' effects remains unclear. Since traditional Chinese medicine has not been widely applied in clinical practice, relevant data are lacking. Furthermore, PC is highly metastatic, so it is of great significance to clarify the detailed procedure $(10,11)$.

\section{Authors' note}

The authors report no conflicts of interest regarding the contents of this work. 


\section{REFERENCES}

1. Yuan SZ. Pancreatic Cancer. Shanghai Science and Technology Press, Shanghai; 2001: 1 .

2. Shi WD, Meng ZQ, Chen Z, Lin JH, Zhou ZH, Liu LM. Identification of liver metastasis-related genes in a novel human pancreatic carcinoma cell model by microarray analysis. Cancer Lett 2009; 283: 84-91.

3. Cheng WW, Liu LM, Yu EX. [Clinical analysis of 202 cases of pancreatic cancer]. Chin J Digest 2003; 23: 758-9.

4. Zhang J, Wang P, Ouyang H, Yin J, Liu A, Ma C, Liu L. Targeting cancer-related inflammation: Chinese herbal medicine inhibits epithelial-to-mesenchymal transition in pancreatic cancer. PLoS One 2013; 8: e70334.

5. Meng Z, Garrett CR, Shen Y, Liu L, Yang P, Huo Y et al. Prospective randomised evaluation of traditional Chinese medicine combined with chemotherapy: a randomised phase II study of wild toad extract plus gemcitabine in patients with advanced pancreatic adenocarcinomas. Br J Cancer 2012; 107: 411-6.

6. Zhang L, Wu C, Zhang Y, Liu F, Zhao M, Bouvet M et al. Efficacy comparison of traditional Chinese medicine LQ versus gemcitabine in a mouse model of pancreatic cancer. J Cell Biochem 2013; 114: 2131-7.

7. Yin JH, Shi WD, Zhu XY, Chen Z, Liu LM. Qingyihuaji formula inhibits progress of liver metastases from advanced pancreatic cancer xenograft by targeting to decrease expression of Cyr61 and VEGF. Integr Cancer Ther 2012; 11: 37-47.

8. Hua YQ, Chen Z, Meng ZQ, Chen H, Shen JG, Wang K et al. High expression of 
erythropoietin-producing hepatoma cell line-B2 (EphB2) predicts the efficiency of the Qingyihuaji formula treatment in pancreatic cancer CFPAC-1 cells through the EphrinB1-EphB2 pathway. Oncol Lett 2014; 8: 17-24.

8. Bex A, Wullich B, Endris V, Otto T, Rembrink K, Stöckle M et al. Comparison of the malignant phenotype and genotype of the human androgen-independent cell line DU145 and a subline derived from metastasis after orthotropic implantation in nude mice. Cancer Genet Cytogenet 2001; 124: 98-104.

9. Feldmann G, Dhara S, Fendrich V, Bedja D, Beaty R, Mullendore M, et al. Blockade of hedgehog signaling inhibits pancreatic cancer invasion and metastases: a new paradigm for combination therapy in solid cancers. Cancer Res 2007; 67: 2187-96.

10. Vincent A, Herman J, Schulick R, Hruban RH, Goggins M. Pancreatic cancer. Lancet 2011; 378: 607-20. 


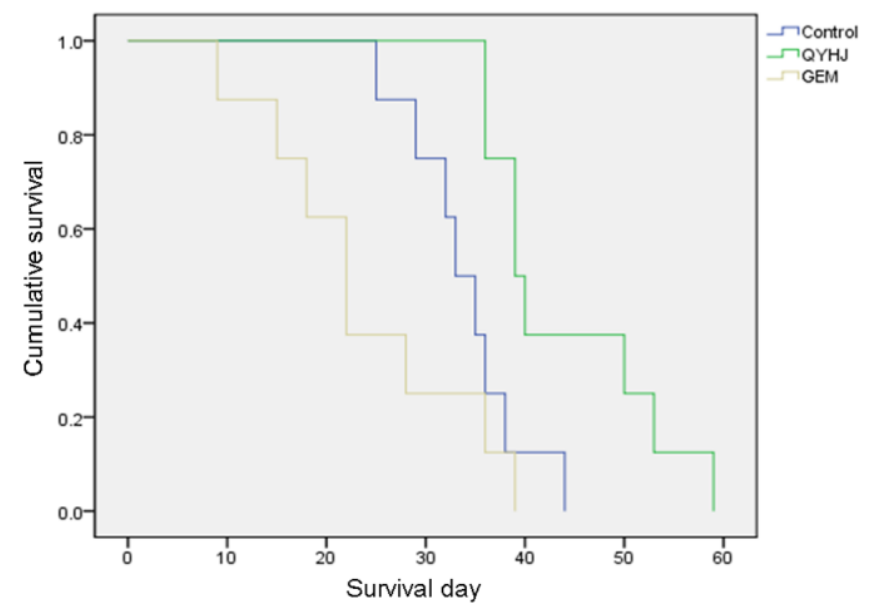

Fig. 1: Survival curves of all mouse groups.

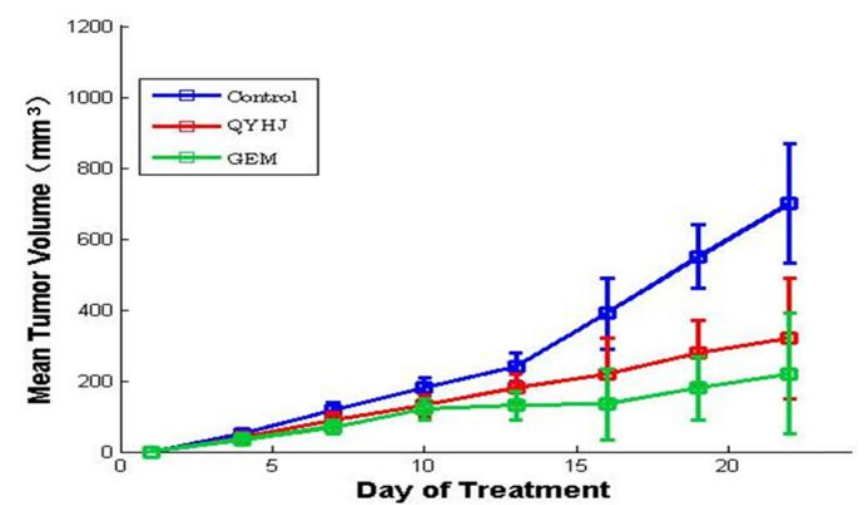

Fig. 2: Tumor growth curves.

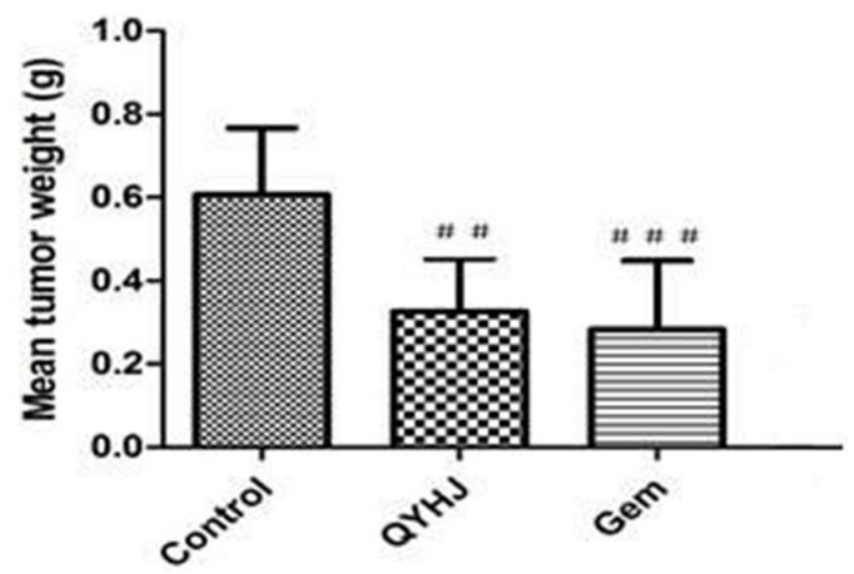

Fig. 3: Inhibition rates of tumors. \#\# $\mathrm{P}<0.01$; \#\#\# $\mathrm{P}<0.001$ (compared with the control group). 


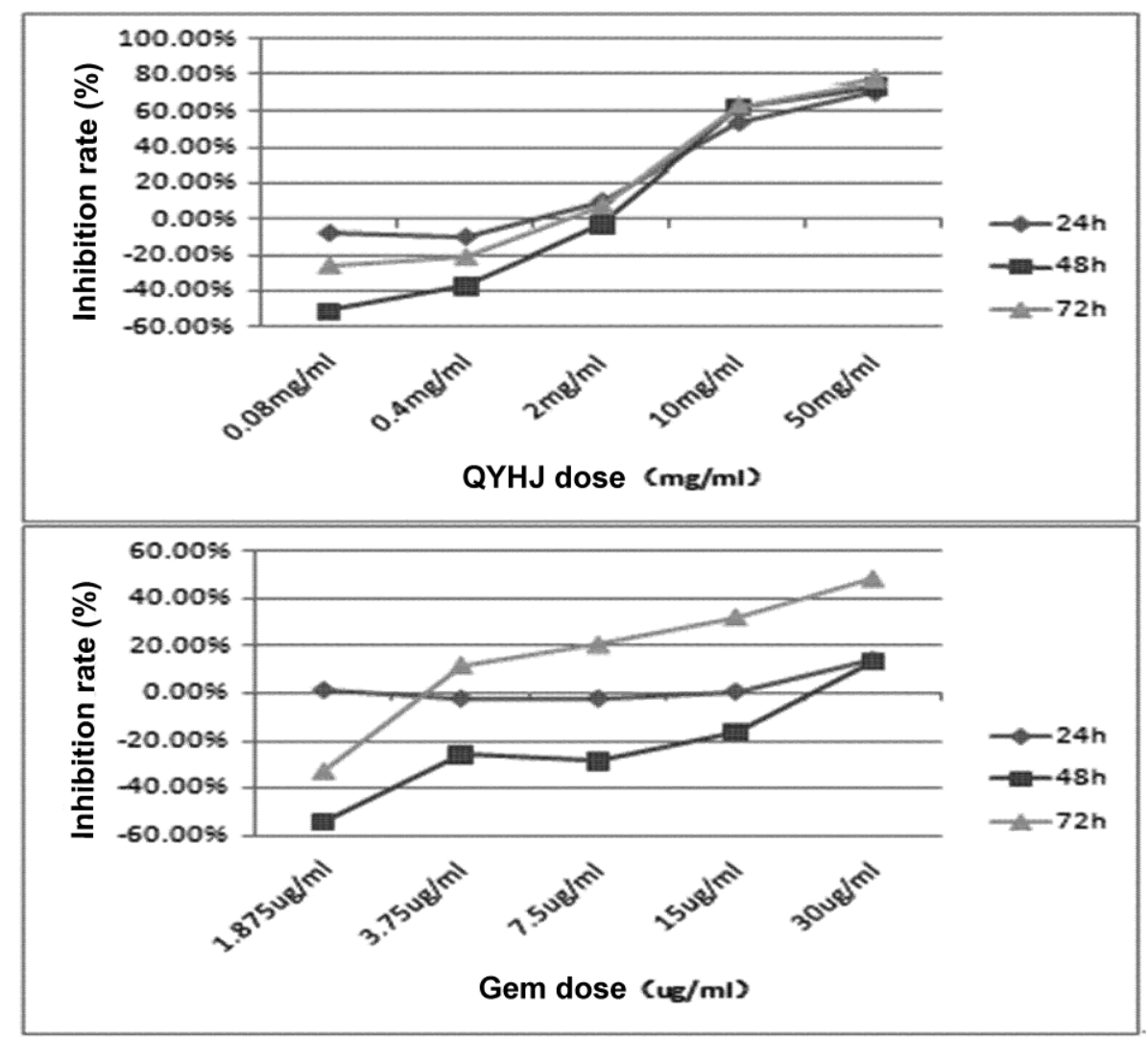

Fig. 4: Inhibition rates of cell proliferation. 